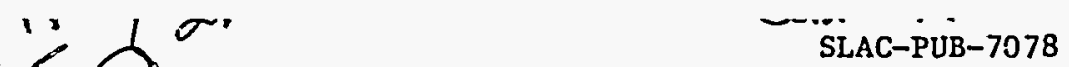
April 1996

\section{Time Profiles and Pulse Structure of Bright, Long Gamma-Ray Bursts Using BATSE TTS Data}

Andrew Lee*, Elliott Bloom ${ }^{*}$ and Jeffrey Scargle ${ }^{\circ}$

-Slanford Linear Accelerator Center, Stanford University, Stanford, CA 94309 - NASA / Ames Research Center, Moffelt Field, CA 94095

\begin{abstract}
The time profiles of many gamma-ray bursts observed by BATSE consist of distinct pulses, which offer the possibility of characterizing the temporal structure of these bursts using a relatively small set of pulse-shape parameters. This pulse analysis has previously been performed on some bright, long bursts using binned data, and on some short bursts using BATSE Time-Tagged Event (TTE) data. The BATSE Time-to-Spill (TTS) burst data records the times required to accumulate a fixed number of photons, giving variable time resolution. The spill times recorded in the TTS data behave as a gamma distribution. We have developed an interactive pulse-fitting program using the pulse model of Norris et al. and a maximum-likelihood fitting algorithm to the gamma distribution of the spill times. We then used this program to analyze a number of bright, long bursts for which TTS data is available. We present statistical information on the attributes of pulses comprising these bursts.
\end{abstract}

\section{BATSE TIME-TO-SPILL DATA}

The BATSE Time-to-Spill (TTS) burst data records the time intervals to accumulate a fixed number of photons, usually 64 photons, in each of four energy channels. Relatively little analysis has been done with the TTS data because it is less convenient to use with standard algorithms than binned data or time-tagged event (TTE) data. However, TTS data offers variable time resolution, ranging from under $50 \mathrm{~ms}$ at low background rates to under $1 \mathrm{~ms}$ in the peaks of the brightest bursts. In contrast, the finest time resolution available for binned data is $16 \mathrm{~ms}$ for the medium energy resolution (MER) data, and then only for the first 33 seconds after the burst trigger. The TTS data usually allows the complete time profiles of bright, long bursts-up to 16,384 spill events (over $10^{6}$ photons) for each channel-to be stored in the limited memory on board the CGRO. This is unlike the TTE data, which is limited to 32,768 photons in all four energy channels combined (see Fig. 1). For short bursts, the TTE data has the advantages of finer time resolution, and of containing data from before the burst trigger time. Some of the shortest bursts are nearly over by the time burst trigger conditions have been met.

*Work supported in part by the Department of Energy contract DE-AC03-76SF00515. Jeffry Scargle acknowledges support from the NASA Astrophysics Data Program.

Presented at the Third Huntsville Gamma-Ray Burst Symposium, Huntsville, Alabama, October 25-27, 1995

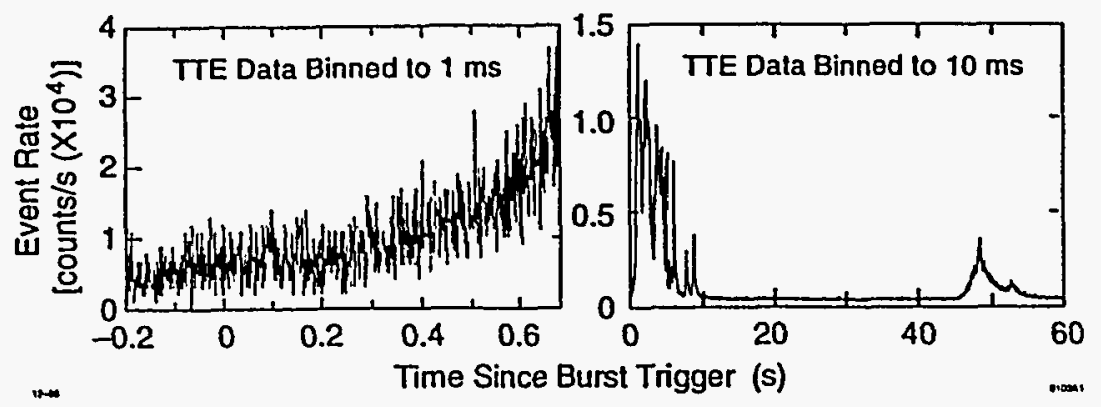

FIG. 1. BATSE Trigger Number 143, a bright, long burst.

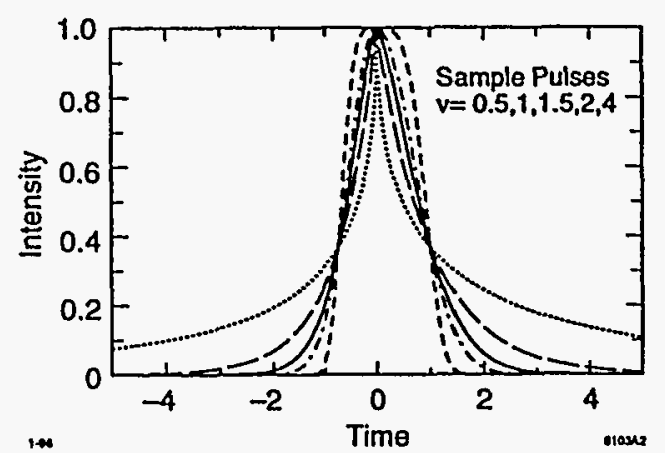

FIG. 2. Sample Pulses, $\nu=0.5,1.0,1.5,2.0,4.0$.

The spill times recorded in the TTS data behave as a gamma distribution, for which the probability of observing a spill time $t$ is

$$
P(t)=\frac{t^{N-1} R^{N} e^{-t}}{\Gamma(N)},
$$

where $N$ is the number of events per spill and $R$ is the individual event rate. This probability distribution is closely related to the Poisson distribution. For large $N$, the gamma distribution approaches a normal distribution, while for $N=1$, it is the exponential distribution for individual time-tagged events.

\section{THE PULSE MODEL}

We have used the phenomological pulse model of Norris et al. to fit burst time profiles. In this model (see Fig. 2), each pulse is described by five parameters with the functional form

$$
I(t)=A \exp \left(-\left|\frac{t-t_{\max }}{\sigma_{r, d}}\right|^{\nu}\right),
$$

where $t_{\max }$ is the time at which the pulse attains its maximum, $\sigma_{r}$ and $\sigma_{d}$ are the rise and decay times, respectively, $A$ is the pulse amplitude, and $\nu$

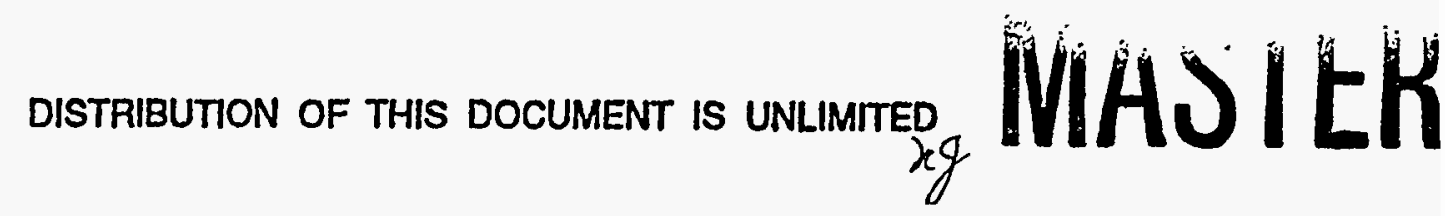




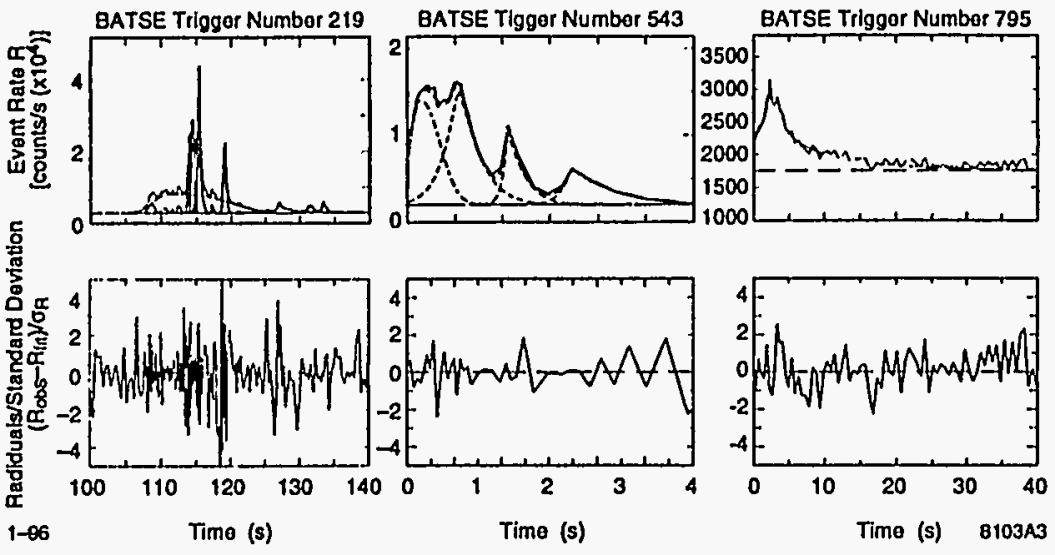

FIG. 3. Gamma-Ray Burst Time Profiles, Energy Cliannel 3, 100-300 keV.

(the "peakedness") gives the sharpness or smoothness of the pulse at its peak. For $\nu=1$, the rise and decay are both simple exponentials, and for $\nu=2$, the rise and decay are Gaussian. Pulses can, and frequently do, overlap.

\section{PULSE-FITTED BURSTS}

We have developed an interactive pulse-fitting program, written in IDL. When fitting pulses to a gamma-ray burst time profile, the user sets the initial pulse parameters, along with the initial parameters for a background with constant slope, graphically. The fitting routine uses a version of the standard IDL routine CURVEFIT, modified to perform a maximum-likelihood fit for the gamma distribution that the TTS spill times follow, rather than the usual $\chi^{2}$ fit. The algorithm used by this routine is the Levenberg-Marquardt gradient-expansion method.

Each spill time in the TTS data file gives the inverse rate at the time of the spill. The program displays the burst time profile in one window, and a second window normally displays,the pulse-fit residuals; that is, the difference between the observed and fitted rates for both the recorded individual spill times and after smoothing. We show only the smoothed data. Instead of the pulse fit residuals, we show the residuals divided by the standard deviation of the data (see Fig. 3).

\section{BURST AND PULSE CHARACTERISTICS}

To date, we have fitted pulses to 109 gamma-ray bursts from the BATSE $2 B$ catalog in energy channel 3, which covers approximately $100-300 \mathrm{keV}$, for a total of 756 pulses. Of these pulses, we considered to be statistically significant only those with amplitudes that differed from zero by at least three standard

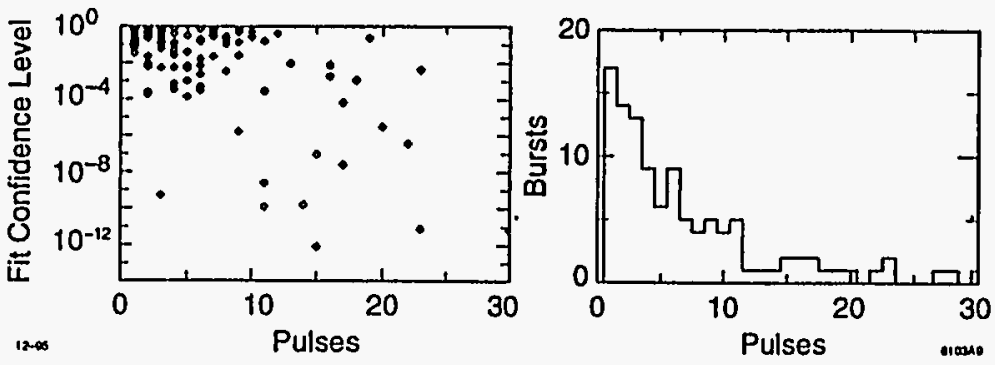

FIG. 4. (left) Fits to complex bursts requiring large numbers of pulses are more likely to have poorer confidence levels.

FIG. 5. (right) Average number of pulses per burst is $6.9 \pm 6.5$; the median is 5 .

deviations, as reported by the fitting routine. Sixteen pulses failed this test, and only the remaining 740 pulses were used to obtain the statistics shown. The results are consistent with those found by Norris et al., fitting all four energy channels to binned BATSE data for 40 gamma-ray bursts. (See Figs. 4 , $5,6$, and 7.$)$

\section{CONCLUSIONS}

This phenomenological pulse model gives an accurate and compact representation of the time profiles of many of the simpler gamma-ray bursts, allowing statistical studies of their characteristics. Some issues arise for more complex bursts that must be fit using many pulses. One issue is the uniqueness of the pulse decomposition when there is large overlap between different pulses. Another is the low confidence levels of many of the fits. It would be possible to obtain fits with higher confidence levels by simply adding more pulses, but this eventually defeats the goal of a compact representation for the burst time profile. In addition, as smaller pulses are added, the statistical significance of the fit parameters decreases, eventually becoming statistically insignificant.
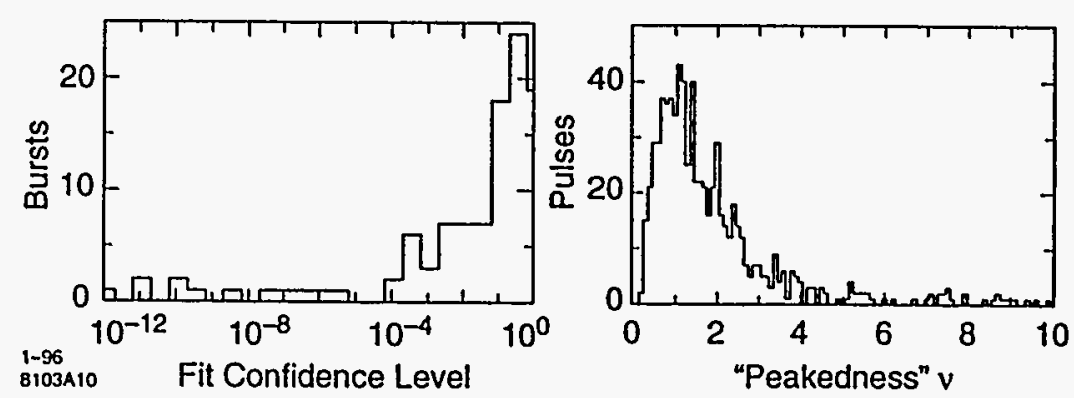

FIG. 6. Average value of "peakedness" paraneter $\nu: 2.0 \pm 1.9$ Median: 1.4. 


\section{DISCLAIMER}

Portions of this document may be illegible in electronic image products. Images are produced from the best available original document. 

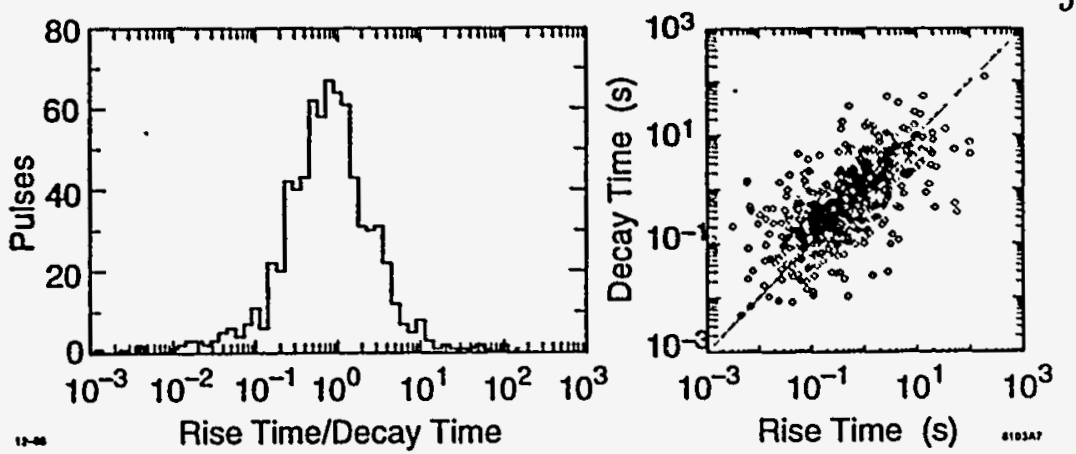

FIG. 7. The ratio of rise to decay times covered a very broad range. The geonctric mean of the ratio is 0.74 with a one standard deviation range of $0.19-2.9$, and the median is 0.77 . (The usual arithunetic mean of the ratio is $2.2 \pm 8.3$, and of the inverse ratio is $5.5 \pm 34$.)

\section{FUTURE WORK}

We plan to complete this pulse analysis for all bright, long BATSE bursts for which TTS data is available, and for all four energy channels, comparing the characteristics of the different energy channels. We will also analyze the pulse-fit residuals to see if they differ from white noise, which would indicate temporal behavior not represented in this pulse model. We will also examine the use of other pulse models.

\section{ACKNOWLEDGMENTS}

This work was supported in part by Department of Energy contract DEAC03-76SF00515. Jeffry Scargle acknowledges support from the NASA Astrophysics Data Program.

\section{REFERENCES}

1. Desai, U.D., Astrophys. Space Sci. 7515 (1981).

2. Fishman, G.J., et al., in Proc. Gamma Ray Observatory Science Workshop, W.N. Johnson, ed. (Greenbelt, MD: NASA/GSFC, 1989) pp. 2-39.

3. Link, B., Epstein, R.I., and Priedhorsky, W.C., ApJ, 408, L81 (1993).

4. Meegan, C.A., et al., BATSE Flight Software User's Manual (Huntsville, AL: NASA/MSFC, 1991)

5. Meegan, C.A., et al., "The Second BATSE Burst Catalog," (Compton Observatory Science Support Center, 1994).

6. Norris, J.P., et al., in Gamma-Ray Bursts - Huntsville, AL 1991, AIP 265, WV.S Pacicsas and G.J. Fishman, eds. (New York: AIP, 1992) p. 294.

7. Norris, J.P., et al., , in Gamma-Ray Bursts-Huntsville, AL 1993, AIP 307, (New York: AIP, 1994) p. 172.

8. Norris, J.P., et al., "Attributes of Pulses in Bright BATSE Gunma-Ray Bursts," submitted to ApJ.

\section{DISCLAIMER}

This report was prepared as an account of work sponsored by an agency of the United States Government. Neither the United States Government nor any agency thereof, nor any of their employees, makes any warranty, express or implied, or assumes any legal liability or responsibility for the accuracy, completeness, or usefulness of any information, apparatus, product, or process disclosed, or represents that its use would not infringe privately owned rights. Reference herein to any specific commercial product, process, or service by trade name, trademark, manufacturer, or otherwise does not necessarily constitute or imply its endorsement, recommendation, or favoring by the United States Government or any agency thereof. The views and opinions of authors expressed herein do not necessarily state or reflect those of the United States Government or any agency thereof. 